adenosine deaminase acting on RNA -1 and -2 (ADAR1; ADAR2). Endothelial cells (ECs) attract and guide leukocytes to sites of ischemic tissue injury. Here we studied the role of RNA editing in ischemic disease.

Methods Primary human and murine vascular endothelial cell cultures were used to assess the EC responses to interleukin-6 (IL-6) or ischemia. For the animal studies, the effect of ADAR2 in acute and chronic ischemic disease was evaluated in cremaster muscle microcirculation by intravital microscopy, in peritoneal cavity after sterile peritonitis and in gastrocnemius muscle after hind-limb ischemia by 8 -colour flow cytometry and immunohistochemistry (IHC) studies of Adar2-/-/tg as well as of i(nducible)EC-ADAR2 knockout (KO) mice. For the mechanistic studies, RNA sequencing, qRT-PCR, western blot, confocal microscopy target-specific miRNA editing studies, RNA-immunoprecipitation, microRNA/plasmid silencing/overexpression and luciferase reporter assays were used. For human studies, ischemic tissues derived from patients with acute or chronic ischemic heart disease were processed.

Results ADAR2, but not ADAR1, expression is induced by $>2$-fold in hypoxic ECs and in ischemic vascular endothelial cells in mice and humans. Unbiased gene ontology analysis of the whole EC transcriptome revealed that ADAR2 controls inflammatory responses and predominantly the expression of interleukin-6-signal transducer (IL6ST), the co-receptor of IL6. Subsequently, ADAR2 controls IL-6 trans-signalling in ECs as documented by STAT3 phosphorylation and expression of the downstream leukocyte adhesion molecules, E-selectin and VCAM-1. IL-6-inflamed cremaster muscles showed that rolling and adhesion of leukocyte subsets to vascular wall were severely impaired in Adar2-/-/tg mice. Leukocyte transmigration was also diminished by $>2$-fold in Adar2-/-/tg and in iEC-ADAR2 KO mice in response to IL-6 or ischemia. Similar results were obtained for leukocyte rolling, adhesion and infiltration after acute $(4 \mathrm{~h})$ and chronic $(3 \mathrm{~d} ; 21 \mathrm{~d})$ ischemia from iEC-ADAR2 KO mice and human ischemic tissues. Next we studied how ADAR2 controls IL6ST expression. ADAR2-deficient EC miRNAome revealed the upregulation of a conserved group of miRNAs targeting the IL6ST mRNA including miR199a-5p and miR-335-3p. In a single nucleotide level, ADAR2-induced RNA editing of the stem loops of the primary miR-199a1/2 and miR-335 directly disrupted Drosha recruitment to both and thus inhibited their maturation process. Accordingly, rescue experiments using miRNA-inhibitors restored IL6ST levels after ADAR2 deficiency.

Conclusion Taking together, inhibition of the microRNA maturation process by ADAR2-mediated RNA editing is integral for IL-6 trans-signalling in vascular endothelium and subsequent leukocyte trafficking to ischemic tissues in mice and humans. Conflict of interest $\mathrm{n} / \mathrm{a}$

\section{BS49 HUMAN EMBRYONIC STEM CELL DERIVED CARDIOMYOCYTES EXPRESS FUNCTIONAL RECEPTORS FOR THE CARDIOVASCULAR PEPTIDE APELIN}

Robyn Macrae*, William G. Bernard, Rhoda E. Kuc, Maria T. Colzani, Thomas Williams, Duuamene Nyimanu, Janet Maguire, Sanjay Sinha, Anthony P. Davenport. University of Cambridge

\subsection{6/heartjnl-2019-BCS.210}

Introduction The apelin receptor is expressed throughout the cardiovascular system, including in cardiomyocytes. Receptor activation by either of its endogenous peptide ligands, apelin or Elabela, has a positive inotropic effect and promotes vasodilatation. Human embryonic stem cell (hESC)-derived cells have the potential for use in translational research to investigate cellular signalling, disease pathogenesis and potential novel treatments. Our aim was to determine if hESC-derived cardiomyocytes express apelin receptor protein and to quantify receptor density to ascertain if this cell type can be used as a phenotypic model for human diseases associated with apelin receptor mutations.

Methods H9 hESCs were cultured to induce differentiation to beating cardiomyocytes. Saturation radioligand binding experiments were performed using [Glp65,Nle75, Tyr77][125I]apelin13 and [Pyr1]apelin-13 to define non-specific binding. Bound radioactivity was counted and data analysed using iterative curve fitting programs to obtain values of receptor affinity (KD) and density (BMAX). Immunocytochemistry was carried out using anti-apelin receptor or anti-cardiac cell marker antibodies.

Results Previous work demonstrated expression of the apelin receptor in hESC-derived cardiomyocytes at the gene level by qRT-PCR at similar levels to adult cells (figure 1A). Here, radioligand binding studies have confirmed receptor protein expression in beating hESC-derived cardiomyocytes. Binding was saturable and [125I]apelin-13 bound with sub-nanomolar affinity $(0.12 \mathrm{nM})$ and receptor density found to be $21 \mathrm{fmol} /$ $\mathrm{mg}$ (figure 1B), comparable to that found in human adult heart. Hill slope was close to 1 consistent with a single binding site for apelin in these cells. Furthermore, beating hESCderived cardiomyocytes stained positive for apelin receptor, in addition to the standard cardiac markers including cardiac troponin $\mathrm{T}$ (figure 2 ).

Conclusion These data importantly confirm that hESC-derived cardiomyocytes express apelin receptor protein at similar levels to adult human heart. Apelin receptor mutations have been identified in the 100,000 Genomes Bridge Project that are associated with rare diseases, including pulmonary arterial hypertension. Our ongoing experiments aim to pharmacologically characterise the apelinergic signalling pathway in the
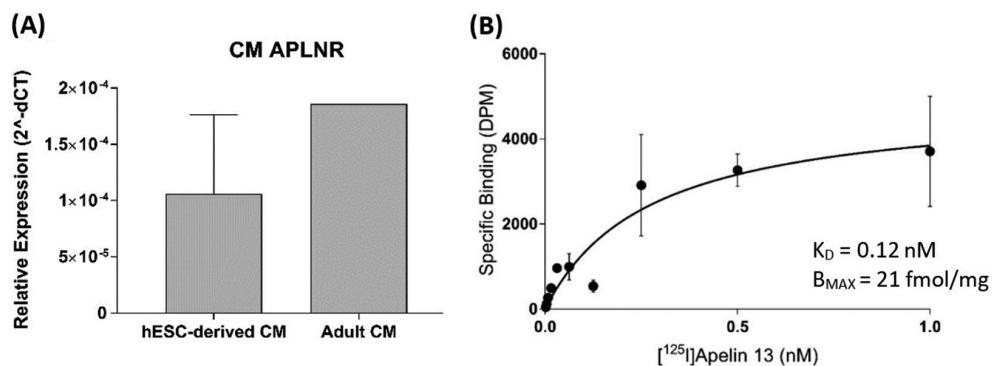

Abstract BS49 Figure 1 (A) Relative expression of apelin receptor (APLNR) gene in hESC-derived cardiomyocytes(CM). Expression is displayed relative to $18 \mathrm{~S}$. (B) Saturation binding curve for beating hESC-derived cardiomyocytes when incubated with increasing concentrations of $\left[{ }^{125}\right.$ I]apelin-13 


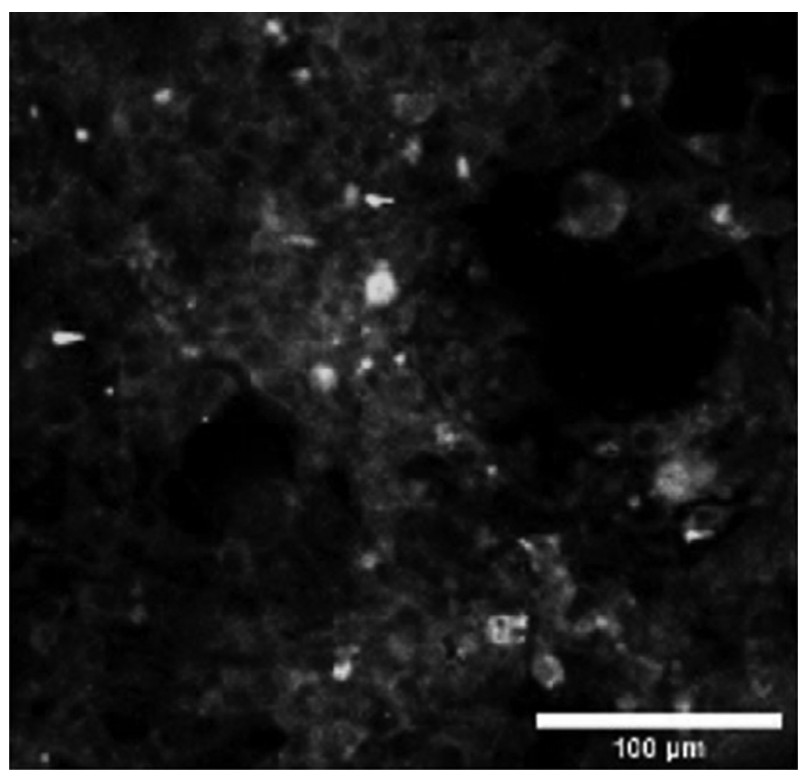

Abstract BS49 Figure 2 Representative image of anti-apelin receptor (green) staining in beating hESC-derived cardiomyocytes. Blue = DAPI, scale bar $=100 \mu \mathrm{m}$

beating hESC-derived cardiomyocytes and we propose to generate hESC-derived phenotypic models by introducing selected apelin receptor mutations via CRISPR/Cas-9 gene editing. Conflict of interest None

\section{BS50 PKC $\alpha$ KNOCK-DOWN INCREASES MEDIAL CALCIFICATION IN A MURINE MODEL OF CHRONIC KIDNEY DISEASE}

${ }^{1}$ Samantha Borland, ${ }^{1}$ Cecilia Facchi* ${ }^{1}$ Julia Behnsen, ${ }^{1}$ Antony Adamson, 'Neil Humphrey, ${ }^{1}$ Michael J Sherratt, ${ }^{1}$ Keith Brennan, ${ }^{2}$ Sheila Francis, 'Nick Ashton, ${ }^{1}$ Ann Canfield. ${ }^{1}$ University of Manchester; ${ }^{2}$ University of Sheffield

\subsection{6/heartjnl-2019-BCS.211}

Medial calcification is the formation of mineralised tissue within the smooth muscle layer of the vessel wall, and frequently occurs in patients with chronic kidney disease. Calcification within the medial layer of the vessel wall can reduce aortic and arterial elasticity, which impairs cardiovascular haemodynamics and results in a significantly elevated risk of morbidity and mortality in the form of hypertension, cardiac hypertrophy and sudden cardiac death. Protein kinase $\mathrm{C} \alpha$ (PKC $\alpha$ ) belongs to the PKC family of serine/threonine kinases and we recently discovered that knocking-down PKC $\alpha$ expression increases high phosphate-induced mineral deposition by vascular smooth muscle cells (VSMCs) in vitro. This study tests the hypothesis that PKC $\alpha$ regulates uraemia-induced medial calcification in vivo.

PKC $\alpha-/-$ mice were generated on the calcification-susceptible DBA/2 background (PKC $\alpha-/-)$ using CRISPR/Cas9 technology. To induce uraemia, wild-type DBA/2 and PKC $\alpha-/-$ mice underwent a two-stage sub-total nephrectomy and were fed a high phosphate $(1.5 \%)$ diet for 8 weeks. Renal function was measured by blood urea nitrogen (BUN). Calcification in the ascending aorta/aortic arch and abdominal aorta were analysed and quantified by micro CT and histology. On average, 68.6

$\pm 3 \%(\mathrm{SD}, \mathrm{n}=3)$ of renal mass was removed from wild-type and $65 \pm 3.2 \% \quad(n=5)$ was removed from PKC $\alpha-/-$ mice $(\mathrm{P}>0.05)$. Loss of $\mathrm{PKC} \alpha$ significantly increased uraemiainduced medial calcification in the abdominal aorta $(-20$-fold increase, $\mathrm{P}<0.05)$ when compared to wild-type controls; there is also a trend for calcification to be increased in the ascending aorta/aortic arch of PKC $\alpha-/-$ mice. Whilst there is a trend for BUN levels to be elevated in PKC $\alpha-/-$ mice $(-1.3$-fold increase compared to wild-type controls, $\mathrm{P}=0.1$ ), there is no correlation between BUN levels and the extent of calcification in these mice.

We have shown previously that inhibiting transforming growth factor- $\beta$ (TGF- $\beta$ ) signalling with SB431542 prevents the increase in calcification observed in PKC $\alpha$-siRNA treated VSMCs. Therefore, to determine the mechanism by which loss of PKC $\alpha$ exerts its effects we examined the relationship between PKC $\alpha$ and TGF- $\beta$ signalling in vitro and in vivo. Our results show that knock-down of PKC $\alpha$ using siRNA increased TGF- $\beta 1$-induced Smad2 phosphorylation in VSMCs in vitro $(\mathrm{P}<0.05)$. Furthermore, phosphorylated Smad2 immunostaining was detected throughout calcified aortic arches from PKC $\alpha-/$ - mice. In contrast, only small areas of phosphorylated Smad2 immunostaining was detected in calcified wild-type controls.

In conclusion, our study suggests that $\operatorname{PKC} \alpha$ may play a protective role in uraemia-induced medial calcification. The PKC $\alpha / T G F \beta$ signalling axis could therefore represent a new therapeutic target for uraemia-induced medial calcification. Conflict of interest None

\section{BS51 AN EVALUATION OF METHODOLOGIES TO INDUCE CALCIFICATION IN VITRO IN HUMAN PRIMARY CELLS}

Sophie Millar*, Susan Anderson, Saoirse O'Sullivan. University of Nottingham

\subsection{6/heartjnl-2019-BCS.212}

Introduction Vascular calcification (VC), the progressive deposition of calcium within the vasculature, is a major risk factor for morbidity, mortality and cardiovascular disease, particularly within chronic kidney disease (CKD) patients. Accurate and reliable in vitro models are essential to allow for investigation into the complex mechanisms behind this extra-osseous biomineralization process. We aimed to investigate the efficacy of a range of media compositions under various conditions (normoxia and hypoxia) to induce calcification in vitro in human

DMEM mineralisation media

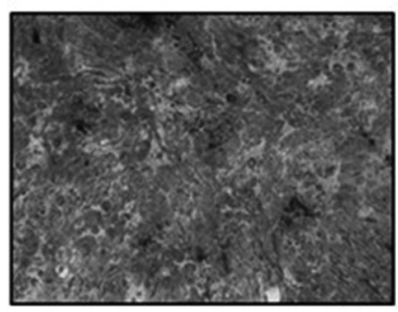

Abstract BS51 Figure 1 Alizarin REd Dye staining after 21 days of culture in DMEM mineralisation media(left) and PromoCell mineralisation media(right) 